\title{
Global Research Highlights
}

Editor's note: CJEM has partnered with a small group of selected journals of international emergency medicine societies to share from each a highlighted research study, as selected monthly by their editors. Our goals are to increase awareness of our readership to research developments in the international emergency medicine literature, promote collaboration among the selected international emergency medicine journals, and support the improvement of emergency medicine world-wide, as described in the WAME statement at http://www.wame.org/about/policy-statements\#Promoting\%20Global\%20Health. Abstracts are reproduced as published in the respective participating journals and are not peer reviewed or edited by CJEM.

\section{Annals of Emergency Medicine}

www.acep.org/annals/

Official journal of the American College of Emergency Physicians (The print version of this article has been scheduled for September 2019)

\section{Emergency Department Management of Out-of-Hospital Laryngeal Tubes}

Brian E. Driver, Sarah K. Scharber, Gabriella B. Horton, Darren A. Braude, Nicholas S. Simpson, Robert F. Reardon

https://doi.org/10.1016/j.annemergmed.2019.01.025

Study objective: Laryngeal tubes are commonly used by emergency medical services (EMS) personnel for out-of-hospital advanced airway management. The emergency department (ED) management of EMS-placed laryngeal tubes is unknown. We seek to describe ED airway management techniques, success, and complications of patients receiving EMS laryngeal tubes.

Methods: Using a keyword text search of ED notes, we identified patients who arrived at our ED with a laryngeal tube from 2010 through 2017. We performed structured chart and video reviews for all eligible patients. In our ED, emergency physicians perform all airway management, and there is no protocol dictating airway management for patients arriving with a laryngeal tube. Using descriptive methods, we report the techniques, success, and complications of ED airway management. Results: We analyzed data on 647 patients receiving out-of-hospital laryngeal tubes, including 472 (73\%) with cardiac arrest from medical causes, $75(21 \%)$ with cardiac arrest from trauma, and $100(15 \%)$ with other conditions. For 580 patients (89\%), emergency physicians exchanged the laryngeal tube for a definitive airway in the ED. Of the 67 patients not intubated in the ED, 66 died in the ED without further airway management. Of the 580 patients intubated in the ED, orotracheal intubation was the first method attempted for 578 (>99\%) and was successful on the first attempt for 515 of 578 (89\%). Macintosh video laryngoscopy (88\% of initial attempts) and a bougie (68\% of initial attempts) were commonly used adjuncts. For 345 of 578 patients (60\%), the laryngeal tube was removed before intubation attempts. For 112 of 578 patients (19\%), the first intubation attempt occurred with the deflated laryngeal tube left in place. Three patients $(<1 \%)$ required a surgical airway.

Conclusion: In this cohort, emergency physicians successfully exchanged an out-of-hospital laryngeal tube for an endotracheal tube, using commonly available airway management techniques. ED clinicians should be familiar with techniques for exchanging out-of-hospital extraglottic airways for an endotracheal tube. 
afjem.com

The official journal of the African Federation for Emergency Medicine, the Emergency Medicine Association of Tanzania, the Emergency Medicine Society of South Africa, the Egyptian Society of Emergency Medicine, the Libyan Emergency Medicine Association, the Ethiopian Society of Emergency Medicine Professionals, the Sudanese Emergency Medicine Society, the Society of Emergency Medicine Practitioners of Nigeria and the Rwanda Emergency Care Association

Fifteen years of emergency medicine literature in Africa: A scoping review

Mould-Millman N, Dixon J, Burkholder TW, Sefa N, Patel H, Yaffeed AQ, Osisanya A, Oyewumi T, Botchey Jr I, Osei-Ampofo M, Sawe H, Lemery J, Cushing T, Wallis LA

Afr J Emerg Med 2019;9(1):14-20

https://doi.org/10.1016/j.afjem.2019.01.006

Introduction: Emergency medicine (EM) throughout Africa exists in various stages of development. The number and types of scientific EM literature can serve as a proxy indicator of EM regional development and activity. The goal of this scoping review is a preliminary assessment of potential size and scope of available African EM literature published over 15 years.

Methods: We searched five indexed international databases as well as non-indexed grey literature from 1999-2014 using key search terms including "Africa", "emergency medicine", "emergency medical services", and "disaster." Two trained physician reviewers independently assessed whether each article met one or more of five inclusion criteria, and discordant results were adjudicated by a senior reviewer. Articles were categorised by subject and country of origin. Publication number per country was normalised by 1,000,000 population.

Results: Of 6091 identified articles, 633 (10.4\%) were included. African publications increased 10-fold from 1999 to 2013 (9 to 94 articles, respectively). Western Africa had the highest number $(212,33.5 \%)$ per region. South Africa had the largest number of articles per country $(171,27.0 \%)$ followed by Nigeria, Kenya, and Ghana. 537 (84.8\%) articles pertained to facility-based EM, $188(29.7 \%)$ to out-of-hospital emergency medicine, and $109(17.2 \%)$ to disaster medicine. Predominant content areas were epidemiology (374, 59.1\%), EM systems $(321,50.7 \%)$ and clinical care $(262,41.4 \%)$. The most common study design was observational (479, 75.7\%), with only $28(4.4 \%)$ interventional studies. All-comers (382, $59.9 \%)$ and children $(91,14.1 \%)$ were the most commonly studied patient populations. Undifferentiated (313, 49.4\%) and traumatic $(180,28.4 \%)$ complaints were most common. Conclusion: Our review revealed a considerable increase in the growth of African EM literature from 1999 to 2014. Overwhelmingly, articles were observational, studied all-comers, and focused on undifferentiated complaints. The articles discovered in this scoping review are reflective of the relatively immature and growing state of African EM.

Reproduced with permission

\section{Emergency Medicine Journal}

emj.bmj.com

Official Journal of the Royal College of Emergency Medicine

\section{Major incident triage and the evaluation of the Triage Sort as a secondary triage method}

James Vassallo, Jason Smith

http://dx.doi.org/10.1136/emermed-2018-207986

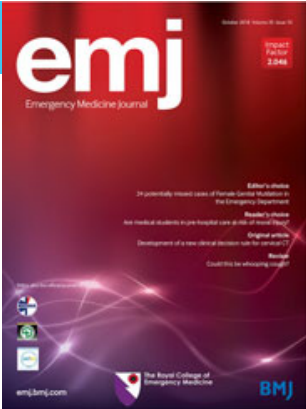

Introduction: A key principle in the effective management of major incidents is triage, the process of prioritising patients on the basis of their clinical acuity. In many countries including the UK, a two-stage approach to triage is practised, with primary triage at the scene followed by a more detailed assessment using a secondary triage process, the Triage Sort. To date, no studies have analysed the performance of the Triage Sort in the civilian setting. The primary aim of this study was to determine the performance of the Triage Sort at predicting the need for life-saving intervention (LSI).

Methods: Using the Trauma Audit Research Network (TARN) database for all adult patients (>18 years) between 2006 and 
2014, we determined which patients received one or more LSIs using a previously defined list. The first recorded hospital physiology was used to categorise patient priority using the Triage Sort, National Ambulance Resilience Unit (NARU) Sieve and the Modified Physiological Triage Tool-24 (MPTT-24). Performance characteristics were evaluated using sensitivity and specificity with statistical analysis using a McNemar's test.

Results: 127233 patients (58.1\%) had complete data and were included: $55.6 \%$ men, aged 61.4 (IOR 43.1-80.0 years), ISS 9 (IOR 9-16), with 24791 (19.5\%) receiving at least one
LSI (priority 1). The Triage Sort demonstrated the lowest accuracy of all triage tools at identifying the need for LSI (sensitivity $15.7 \%$ (95\% Cl 15.2 to 16.2$)$ correlating with the highest rate of undertriage $(84.3 \%(95 \% \mathrm{Cl} 83.8$ to 84.8$)$, but it had the greatest specificity $(98.7 \%$ (95\% Cl 98.6 to 98.8$)$.

Conclusion: Within a civilian trauma registry population, the Triage Sort demonstrated the poorest performance at identifying patients in need of LSI. Its use as a secondary triage tool should be reviewed, with an urgent need for further research to determine the optimum method of secondary triage.

\section{Emergencias}

emergencias. portalsemes.org/English

\section{Official Journal of the Spanish Society of Emergency Medicine}

\section{Differences in emergency department care of adults with a first epileptic seizure versus a recurrent seizure: a study of the ACESUR (Acute Epileptic Seizures in the Emergency Department) registry}

Cesáreo Fernández Alonso, Raúl Alonso Avilés, Manuel Liñán López, Félix González Martínez, Manuel Fuentes Ferrer, Belén Gros Bañeres, en representación del grupo ACESUR

Cited: Cesáreo Fernández Alonso, Raúl Alonso Avilés, Manuel Liñán López, Félix González Martínez, Manuel Fuentes Ferrer, Belén Gros Bañeres, en representación del grupo ACESUR

http://emergencias.portalsemes.org/descargar/registro-acesur-atencin-de-pacientes-adultos-con-crisis-epilpticas-en-servicios-deurgencias-diferencias-entre-primer-episodio-y-recurrencia/

Objective: To describe the characteristics of care received by patients who come to the emergency department with a first epileptic seizure versus a recurrent seizure in a patient with diagnosed epilepsy.

Methods: ACESUR (Acute Epileptic Seizures in the Emergency Department) is a prospective multicenter, multipurpose registry of cases obtained by systematic sampling on even days in February and July 2017 and on odd days in April and October 2017. Patients were aged 18 years or older and had an emergency department diagnosis of epileptic seizure. We recorded clinical variables and details related to care given during each patient's visit, including whether the event was a first or recurrent seizure.

Results: A total of 664 patients attended by 18 Spanish emergency departments were entered into the ACESUR registry. Two hundred twenty-nine (34.5\%) were first seizures and $435(65.5 \%)$ were recurrences. Patients who were attended for first seizures were older, consulted for a wider variety of reasons, and were transported in ambulances $(P<.001$, all comparisons). Care received differed between patients with first seizures versus recurrent seizures. Specific complementary testing was more likely in patients with first seizures (adjusted odds ratio [aOR], 13.94; 95\% Cl, 29-26.7; $\mathrm{P}<.001$ ), and they were more often hospitalized or stayed longer in the emergency department, (aOR, 1.69; 95\% Cl, 1.11-2.58; $\mathrm{P}=.015$ ). Pharmacologic treatment did not differ between the groups, either in the acute phase or for prevention (aOR, 1.40; 95\% $\mathrm{Cl}, 0.94-2.09 ; \mathrm{P}=.096)$. Antiepileptic drugs were given to 100 patients $(43.7 \%)$ after a first seizure and were restarted or changed in 142 patients with recurrent seizure (32.6\%).

Conclusion: The clinical characteristics of adults attended for a first epileptic seizure differ from those of patients with diagnosed epilepsy who were attended for recurrent seizures in Spain. The care received also differs. 


\section{Hong Kong Journal of Emergency Medicine}

hkjem.com

Official Journal of the Hong Kong College of Emergency Medicine

\section{Is lung ultrasonography a useful method to diagnose children with community-acquired pneumonia in emergency settings?}

Aykut Çağlar, Emel Ulusoy, Anıl Er, Fatma Akgül, Hale Çitlenbik, Durgül Yılmaz and Murat Duman

Background: Lung ultrasonography is a new method for diagnosing community-acquired pneumonia.

Lung ultrasonography has some advantages over chest X-ray, such as lack of ionizing radiation risk,

bedside performance, and cost-effectiveness.

Objective: In this study, we aimed to determine the feasibility of lung ultrasonography in emergency

settings in children with community-acquired pneumonia.

Methods: The study included patients younger than 18 years of age with suspicion of community-acquired pneumonia. On the first evaluation, patients with positive clinical and/or chest $X$-ray findings were defined to have community-acquired pneumonia, and this was accepted as the gold standard. The chest $X$-rays were evaluated by the chief of the pediatric emergency department, who was blinded to the patients and the lung ultrasonography results. Lung ultrasonography was performed by another pediatric emergency physician who was also blinded to the chest $X$-ray results and clinical findings such as fever, respiratory distress, rales, and wheezing.

Results: Of the 91 patients enrolled, 71 (78.0\%) were diagnosed with community-acquired pneumonia based on clinical and chest $X$-ray findings. The median (interquartile range) duration of the lung ultrasonography procedure was 4.0 (3.5-6.0) min. Shred sign, air bronchogram, and hepatization were significantly more frequent in the patients with community-acquired pneumonia $(p<0.01, p<0.01$, and $p=0.01$, respectively). Sensitivity and specificity of lung ultrasonography were $78.5 \%(67.1-87.4)$ and 95.2\% (76.1-99.8), respectively.

Conclusion: Lung ultrasonography is a useful diagnostic method for children with suspicion of community-acquired pneumonia. 